\section{KBV-AG uneinig: Laborhonorar bleibt vorerst rechtswidrig}

_ Auf Beschluss der KBV-Vertreterversammlung sucht eine Arbeitsgruppe (AG) nach einer besseren Grundlage für die Vergütung labormedizinischer Leistungen. Die bisherige Vorgehensweise gilt als rechtswidrig, da Laborleistungen

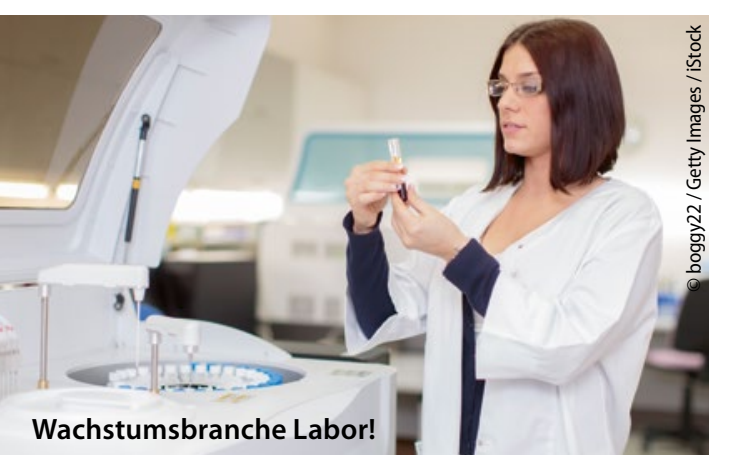

einschließlich Wirtschaftlichkeitsbonus nach KBV-Vorgabe aus einem gesonderten „Grundbetrag Labor“ vergütet werden. Reicht das dort eingestellte Geld nicht aus, muss aus der Gesamtvergütung zugeschossen werden, und zwar in gleicher Höhe aus dem haus- und dem fachärztlichen Honoraranteil.

Dieser Mechanismus verstößt nach einer Stellungnahme des Bundesgesundheitsministeriums vom 2. Mai 2016 gegen den Trennungsbeschluss im Versorgungsstrukturgesetz, wonach hausärztliche Leistungen aus dem Hausarztund fachärztliche aus dem Facharzttopf der Gesamtvergütung zu honorieren sind. Da der größte Teil des Laborhonorars im fachärztlichen Bereich angefor-

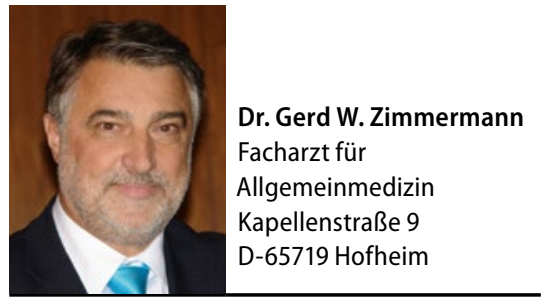

dert wird, finanzieren die Hausärzte fachärztliche Leistungen mit.

\section{MMW-KOMMENTAR}

Die sechs Hausärzte und sechs Fachärzte der AG "Weiterentwicklung Labor" konnten sich bisher nur auf Stückwerk einigen (siehe Meldung rechts), nicht auf eine grundlegende Reform. In einigen KVen wurden deshalb bereits Muster-Klageverfahren eingeleitet. Voraussichtlich werden die Richter des Bundessozialgerichts (BSG) für eine Entscheidung sorgen müssen. Widerspruch gegen die Honorarbescheide muss der einzelne Hausarzt nicht unbedingt einlegen: Im Falle einer positiven Entscheidung des BSG müssen die KVen ihre Honorarverteilung anpassen und alle Honorarbescheide neu fassen.

\title{
Delegationsleitsungen im EBM neu geordnet
}

_ Seit dem 1. Juli 2016 gibt es neue Leistungen für den Einsatz von Praxispersonal bei Haus- oder Heimbesuchen. Die bisherigen Nrn. 40240 und 40260 EBM für ärztlich angeordnete Hilfeleistungen von Praxismitarbeitern werden durch die Nrn. 38100 (7,93 Euro) und 38105 (4,07 Euro) ersetzt und können für

\section{Tab. 1 Die neuen delegierbaren Besuchsleistungen}

\begin{tabular}{|l|l|l|}
\hline EBM & Beschreibung & Euro \\
\hline 38100 & MFA-Besuch & 7,93 \\
\hline 38105 & MFA-Besuch bei weiteren Patienten in derselben sozialen Gemeinschaft & 4,07 \\
\hline $\begin{array}{l}38100 \\
38200\end{array}$ & NäPa-Besuch im Alten- oder Pflegeheim & 17,32 \\
\hline $\begin{array}{l}38105 \\
38205\end{array}$ & NäPa-Besuch bei weiteren Patienten im selben Heim & 12,73 \\
\hline 03062 & NäPa-Besuch (Praxis erreicht Mindestpatientenzahl) & 17,32 \\
\hline 03063 & $\begin{array}{l}\text { NäPa-Besuch bei weiteren Patienten in derselben sozialen Gemeinschaft } \\
\text { (Praxis erreicht Mindestpatientenzahl) }\end{array}$ & 12,73 \\
\hline berechenbar je Sitzung, inklusive Wegekosten & \\
\hline
\end{tabular}

Besuchsleistungen von medizinischen Fachangestellten (MFA) berechnet werden. Bei Besuchen durch besonders qualifizierte nichtärztliche Praxisassistenten (NäPa) kann das Honorar mittels der Zuschlagsziffern 38200 bzw. 38205 auf 17,32 bzw. 12,73 gesteigert werden. Diese Leistungen sind auf Besuche in Alten- und Pflegeheimen beschränkt.

\section{MMW-KOMMENTAR}

Die NäPa-Leistungen nach den Nrn. 03062 und 03063 können weiterhin berechnet werden - auch für Besuche, die nicht in Heime führen. Allerdings muss man eine Mindestzahl an Behandlungsfällen je Quartal nachweisen: 860 für eine Einzelpraxis, 640 für jeden weiteren Arzt in einer Gemeinschaftspraxis, alternativ 160 bzw. 120 Patienten im Alter von mindestens 75 Jahren. 